\title{
Spatial transition management for improving outdoor cinematic augmented reality experience of the TV show
}

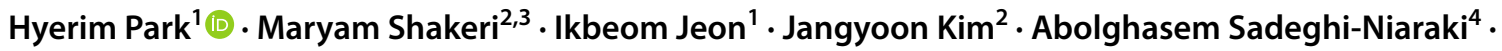 \\ Woontack Woo ${ }^{1,2}$ (1)
}

Received: 9 July 2021 / Accepted: 14 December 2021 / Published online: 6 January 2022

(c) The Author(s), under exclusive licence to Springer-Verlag London Ltd., part of Springer Nature 2022

\begin{abstract}
There have been attempts to provide new cinematic experiences by connecting TV or movie content to suitable locations through augmented reality (AR). However, few studies have suggested a method to manage breakdowns in continuity due to spatial transitions. Thus, we propose a method to manage the spatial transition that occurs when we create a TV show trajectory by mapping TV show scenes with spatiotemporal information to the real world. Our approach involved two steps. The first step is to reduce the spatial transition considering the sequence, location, and importance of TV show scenes when creating the TV show trajectory in the authoring tool. The second is to fill the spatial transition with additional TV show scenes considering sequence, importance, and user interest when providing the TV show trajectory in the mobile application. The user study results showed that reducing spatial transition increases narrative engagement by allowing participants to see important content within the trajectory. The additional content in spatial transition decreased the physical demand and effort in terms of the perceived workload, although it increased the task completion time. Integrated spatial transition management improved the overall cinematic augmented reality (CAR) experience of the TV show. Furthermore, we suggest design implications for realizing the CAR of TV shows based on our findings.
\end{abstract}

Keywords Cinematic augmented reality $\cdot$ Augmented reality $\cdot$ Trajectory $\cdot$ Continuity

\section{Introduction}

Cinematic augmented reality (CAR) is a comprehensive concept of dramatic or narrative experiences in an AR environment. It includes passive locative media as well as participating in interactive storytelling, based on various devices such as public screens, ${ }^{1}$ projectors (Mine et al. 2012), wearable devices (MacIntyre et al. 2002), and mobile devices (Tung

Woontack Woo

wwoo@kaist.ac.kr

1 KAIST UVR Lab, \#2325, N5, 291, Daehak-ro, Yuseong-gu, Daejeon, Republic of Korea

2 KAIST KI-ITC Augmented Reality Research Center, \#303, E4, 291, Daehak-ro, Yuseong-gu, Daejeon, Republic of Korea

3 Geodesy \& Geomatics Engineering, K.N. Toosi University of Technology, Tehran, Iran

4 Dept. of Computer Science and Eng., and Convergence Eng. for Intelligent Drone, Sejong University, Seoul, Republic of Korea
2015). There have been various attempts to provide narrative experiences using AR technology. For example, indoors, MacIntyre et al. provided a different character's point of view and interpretation of the classic television play according to the character's position (MacIntyre et al. 2003). Players can also interact with artificial intelligence-based virtual characters of the AR interactive drama (Dow et al. 2006). Outdoors, users visited various places along the linear narrative (Wither et al. 2010) or participated in the conversational storytelling by interacting with virtual characters (Spierling and Kampa 2014). However, most attempts have been made to provide a fragmented narrative experience in a single place (Park and Woo 2017) or a newly created locative storytelling based on multiple locations (Hansen et al. 2012; Shin et al. 2017).

There has been no attempt to map a movie or TV show's content to multiple locations and provide a continuous cinematic experience. Various issues arise when sequentially delivering and experiencing a TV show's story based on

\footnotetext{
$\overline{1}$ https://www.behance.net/gallery/33549335/Worlds-First-Cinem atic-Augmented-Reality-Experience.
} 
the real world. It is challenging to generate a feasible and practical tour path when exploring space in the order of the story (Torchin 2002). This is because, in many cases, the background of the next scene in the story is physically distant, or non-continuous scenes are concentrated in a specific background location. It creates a zigzagging path or makes visitors double back (Packer et al. 2017). In addition, users can experience various internal and external distractions that interfere with the continuity of the narrative experience (Benford et al. 2009). For instance, users can feel mental or physical workload when exploring a 3D environment to view the distributed content. They can also encounter other compelling content or places that break the continuity of the coherent narrative experience. These issues are mainly due to spatial transition when content with spatial and temporal contextual information is mapped to the real world.

We propose spatial transition management to provide a continuous and coherent cinematic experience of a TV show in AR. Spatial transition means that the user physically moves to another place to view the content. We managed the spatial transition in two steps. First, we reduced the spatial transition by considering the sequence, location, and importance of TV show content when creating the TV show trajectory. It aims to provide important TV show content sequentially within a simple tour path. Second, we filled the spatial transition with additional TV show content taking into account the order and importance of content and users' interests. We expected that it would prevent continuity breakdowns and maintain narrative engagement while moving. For the user study, we implemented a desktop-based authoring tool and mobile application. In the user study, we compared the three trajectories: (1) basic trajectory, (2) summarization trajectory, and (3) additional summarization trajectory. The basic trajectory participants experienced a TV show trajectory created by considering only the sequence of TV show scenes. The summarization trajectory participants experienced a TV show trajectory with reduced spatial transitions. The additional summarization trajectory participants experienced a TV show trajectory, viewing supplementary TV show content when moving to the next location.

The results showed that reducing spatial transition increases narrative engagement by allowing participants to see important content within the trajectory. The additional content in spatial transition decreased the physical demand and effort in terms of the perceived workload, although it increased the task completion time. Integrated spatial transition management increased the narrative engagement and subscales such as narrative understanding and attentional focus. It decreased the overall perceived workload and subscales such as physical demand, temporal demand, and effort. It also increased the evaluation of system usability. From the user study results, we demonstrated that the proposed spatial transition management significantly enhanced the cinematic AR experience of the TV show. Finally, we suggest a design implementation to realize CAR for TV shows based on our findings. The main novel contributions of the present study are as follows:

- We introduce the concept of TV show trajectory and propose the spatial transition management.

- We prove the significant effect of spatial transition management on CAR experience through a user study.

- We present design implications to realize the CAR of TV shows based on our experimental results.

\section{Related works}

Benford et al. proposed the concept of a trajectory as a journey through user experience (Benford and Giannachi 2008; Benford et al. 2009, 2011). They emphasized its overall continuity and coherence as all user experiences occur continuously based on time and space. They argued that various transitions of space, time, roles, and interfaces should be addressed to manage continuity when experiencing digital media trajectories based on the physical world. However, studies that have adopted this concept into the systems or user studies have focused on managing engagement in local trajectories (Fosh et al. 2013; Kim et al. 2018a). Although there are more dynamic transitions in a global trajectory connecting local trajectories, there is little research on managing and maintaining user engagement on a global trajectory.

Various attempts have been made to create and provide locative storytelling (Liestøl 2014; Nisi et al. 2017; Hargood et al. 2017). StoryPlaces offers novel functions to manage location, content, and geographic and temporal conditions (Hargood et al. 2018). The cyber-cartographic application (Caquard and Fiset 2014) was designed to help appropriately map various dimensions of narratives. Naliuka et al. presented a general-purpose narrative architecture that allows a considerable range of story-based games and guide content to be expressed in a location-aware manner (Naliuka et al. 2010). However, these studies have mainly focused on crafting new stories from the author's point of view. Few studies have proposed a method to provide a locative narrative experience of existing content with spatiotemporal information. In addition, few studies have systematically examined user experiences in locative narratives. Packer et al. created guidelines for managing spatial transitions through user interviews of locative fiction (Packer et al. 2017). However, few studies have applied these guidelines and investigated their effectiveness through user studies.

Recently, attempts have been made to provide new cinematic experiences by connecting TV or movie content to suitable locations. For example, Ghellal et al. remediated "Nosferatu," a German expressionist horror movie, to the 
location-based AR horror adventure (Ghellal et al. 2014). They created hybrid spaces by merging the original movie clips and suitable locations. They allowed users to watch the location-based content freely and to make their own trajectories of the fictional world. Park et al. proposed the concept of location-based film experiences (Park and Woo 2015). They enabled users to search and watch a video clip based on the filming locations through VR (Park et al. 2018b) and AR application (Park and Woo 2017; Park et al. 2018a). Nevertheless, these studies provided fragmented content experiences in a single PoI. They did not provide a systematic method for generating and providing an continuous and coherent cinematic experiences based on multiple places.

Therefore, we proposed a method to map existing TV show content to the real world from the perspective of spatial transition management. We verified the effects of the proposed method on the cinematic experience of users through a field study.

\section{Method}

This section introduces the concept of a TV show trajectory and explains how to manage spatial transitions. It also details the system implementation and setup for the user study.

\subsection{Concept of TV show trajectory}

The trajectory is an $\mathrm{HCI}$ concept that emphasizes the overall continuity and coherence of the user experience when users experience digital media based on the physical world. In this study, we applied the concept of trajectory to the design of the CAR experience using a TV show. The TV show trajectory aims to sequentially provide $\mathrm{TV}$ show content to people who visit the TV show's background. We expected that it would enable people to experience a narrative world of TV shows through an AR environment.

As shown in Fig. 1, the TV show trajectory consists of AR content based on TV show scenes related to the visiting places. In broadcasting video resources, a video usually denotes episodes, and an episode contains sequences, scenes, shots, and frames in a hierarchical structure (Hunter and Armstrong 1999). The episode is a collection of sequences packaged for distribution. The sequence is a collection of scenes, a series of situations or events occurring at the same time and place. The scene is a basic story unit used in a script and a call sheet ${ }^{2}$ of a TV series. As shown in Fig. 1, an episode consists of a number of scenes, and the episodes and scene numbers are specified in the script. We can detect the scene and episode changes by comparing the script and the

\footnotetext{
${ }^{2}$ A daily schedule of filming for a movie or television show.
}

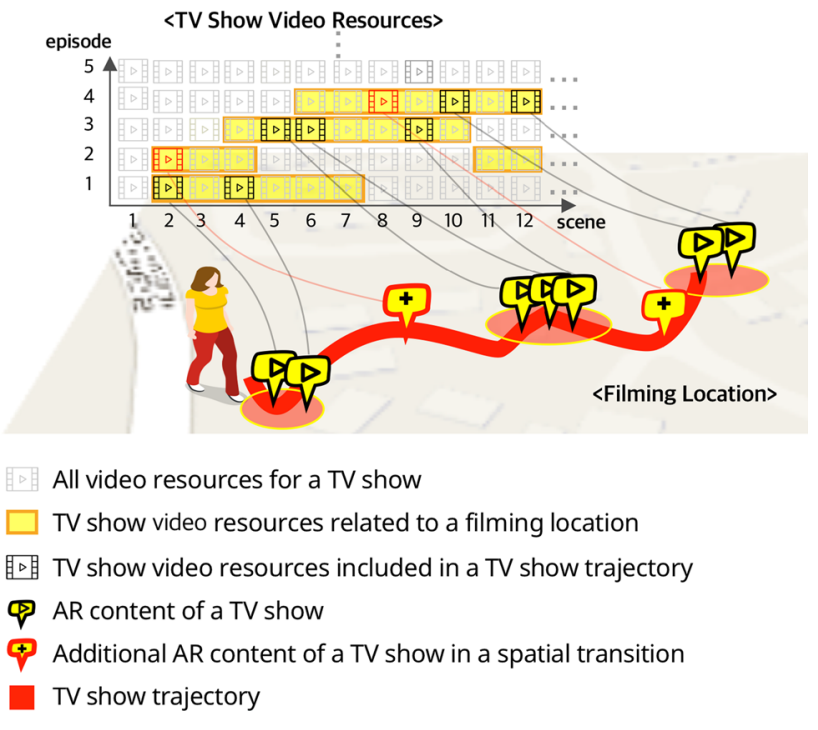

Fig. 1 Concept of TV show trajectory in cinematic augmented reality

video source. Thus, we selected the "scene" of the TV show as the TV show trajectory's basic content unit. As a scene is the basic unit of a TV show trajectory, we can provide a TV show story based on location and easily obtain contextual data about the scene (e.g., characters, places, time, props, etc.) from a script or call sheet.

However, it is almost impossible to sequentially experience the story of a TV show based on real places. This is because spatial transitions occur when content containing spatial and temporal contextual information is mapped to the real world. When scenes are mapped to actual background locations, users have to visit distant places to view the next scene or constantly revisit the story's main place. Therefore, a TV show trajectory that does not consider spatial transitions creates impractical and unrealistic CAR routes. Consequently, we propose a method for managing spatial transitions when generating and providing TV show trajectories to provide an engaging cinematic experience of TV shows in AR environments.

\subsection{Spatial transition management}

Our approach involved two steps. The first is to reduce spatial transitions in creating a TV show trajectory in the authoring tool. The second is to fill the spatial transition with additional content when providing the TV show trajectory in the mobile application.

\subsubsection{Reducing the spatial transition}

To reduce the spatial transition of the trajectory, we recommended content to authors by considering the order, location, and importance of the TV show scene. By considering both 
the order and location of the TV show scene, we expected to improve the inefficient path for viewing scattered content. In addition, we attempted to provide an essential story in the trajectory by considering the content importance.

Episode and scene numbers are specified in the script and call sheet. These are temporal information that provide TV show's AR content in order. We represent the TV show scene's AR content as $a$, the episode number as $i$, and the scene number as $j$. When AR content $a_{i j}$ has been authored in the TV show trajectory according to the author's intention, we recommend the next content as follows. We denote the next AR content of $a_{i j}$ as $a_{m n} . m$ is the number of episodes, and $n$ is the number of scenes.

$w_{m n}=\frac{c_{m n}}{d_{m n}} \quad$ for $\left\{\begin{array}{c}m=i, \quad n>j \\ m>i, \quad n^{\forall}\end{array}\right.$

The weight $w_{m n}$ is calculated by content importance $c_{m n}$ and the distance $d_{m n}$ for AR content $a_{m n}$ that meets the assumptions of $m$ and $n$, as shown in Eq. 1. The condition of the episode number and scene number provides content in the order of the story. The subject for recommendation must be scenes after $a_{i j}$. Thus, within the same episode $(m=i)$, the scene number $n$ must be greater than $j$. In subsequent episodes $(m>i)$, all scenes $\left(n^{\forall}\right)$ are subjects for the recommendation. The previous scenes of $a_{i j}$ were not considered for recommendation.

$c_{m n}=\operatorname{play}\left(a_{m n}\right)+\operatorname{like}\left(a_{m n}\right)$

$c_{m n}$ implies the importance of $a_{m n}$. It is calculated as the sum of play $\left(a_{m n}\right)$ and like $\left(a_{m n}\right)$, as shown in Eq. 2. We propose that the more people view and like, the more important is the content. play $\left(a_{m n}\right)$ denotes the number of times users play AR content $a_{m n}$ and like $\left(a_{m n}\right)$ represents how many times users push the like button of AR content $a_{m n}$. However, when we first implemented the system, there was no data on the importance of AR content. Therefore, we calculated importance based on the views and likes of video clips containing the scene in a YouTube official channel operated by the broadcaster. For each scene, the view and like numbers were normalized by the maximum number of views and likes in our dataset, respectively.

$d_{m n}=\left\|\operatorname{pos}\left(a_{m n}\right)-\operatorname{pos}\left(a_{i j}\right)\right\|$

$d_{m n}$ is the distance and is calculated by using two GPS points, $\operatorname{pos}\left(a_{m n}\right)$ and $\operatorname{pos}\left(a_{i j}\right)$, in the metric units in the 3D Cartesian coordinate system, as shown in Eq. 3. The weight $w_{m n}$ aims to provide $a_{m n}$ that maximizes the content importance and minimizes the distance from the previous AR content. When the author selects the content from a recommended content list, the system recommends the following content based on the above method. When the author completes a local trajectory, the scenes for that place are excluded from the recommendation to prevent re-visiting the site.

\subsubsection{Filling the spatial transition}

To fill the spatial transition in the authored trajectory, we provided additional content to the users. It takes into account the story sequence and importance and user interests. We expected the proposed method to prevent disengagement by spatial transitions and to keep users immersed in the TV show narrative on the go.

$$
\begin{array}{r}
r_{p q}=\arg \max _{a_{p q}}\left(w_{p q}\right) \quad \text { for } \quad m \geq p \geq i, \\
\text { if } \begin{cases}p=i, & q>j \\
p=m, & q<n\end{cases}
\end{array}
$$

We provide the AR content $r_{p q}$ with the largest weight $w_{p q}$ among $a_{p q}$, as shown in Eq. 4. $a_{p q}$ is AR content that satisfies the condition of episode number $p$ and scene number $q$ when there is a spatial transition between consecutive AR content $a_{i j}$ and $a_{m n} . a_{i j}$ is the AR content that the user visited, and $a_{m n}$ is the next AR content to be visited. The additional content in the middle of moving to view $a_{m n}$ should be the AR content between $a_{i j}$ and $a_{m n}$.

Thus, the episode number $p$ should be greater than $i$ and does not exceed $m$. If the episode number $p$ is the same as the episode number $i$, the scene number $q$ must be greater than $j$. If the episode number $p$ is the same as the episode number $m$ of the next AR content, the scene number $q$ must be less than $n$. The spatial transition is recognized based on a different place tag contained in $a_{i j}$ and $a_{m n}$.

$w_{p q}=\frac{1}{G} \sum_{e=1}^{G} f_{s}\left(h_{e}^{u}, a_{p q}\right)+c_{p q}$

The weight $w_{p q}$ is calculated by summing the value of normalizing the sum of tag similarity $s$ and the content importance $c_{p q}$ of $a_{p q}$, as shown in Eq. 5. The tag similarity $s$ is used to recommend content by reflecting the user's content interest. The function for tag similarity $f_{s}$ compares the tag instance between all AR content $h_{e}^{u}$ that users have played and AR content $a_{p q}$ which is the subject for the recommendation. It is calculated as 1 if the tag instance is equal, and 0 otherwise.

As shown in Table 1, we organized the tag information by adopting a $\mathrm{W}^{4}$ (i.e., who, when, where, and what) information structure. This layout is used to consider the integrated context of location-based content for point-of-interest recommendations (Gao et al. 2015). We generated tag information when creating AR content in the authoring tool by referencing the call sheet and script. We used these data to consider the spatiotemporal information of the TV show content and estimate user interests. 
Table 1 Tags of AR content of TV show

\begin{tabular}{ll}
\hline & Tag category \\
\hline who & actor \\
when & time span, time \\
where & place \\
what & genre, title, description, \\
& episode number, scene \\
& number \\
\hline
\end{tabular}

$c_{p q}$ is calculated in the same way as in Eq. 2. The recommended content $r_{p q}$ is augmented at the midpoint of the path between the AR content $a_{i j}$ and $a_{m n}$.

\subsection{User study}

We designed a user study to analyze the effect of the proposed method on the CAR experience of the TV show. To conduct user studies, we implemented desktop-based authoring tools for creating TV show trajectories and mobile applications for providing TV show trajectories on-site. We selected a representative TV show for film-induced tourism. We collected and produced the AR content of the TV show to create a TV show trajectory according to the experimental conditions. We evaluated the user experience of each trajectory using quantitative and qualitative evaluation. The following sections describe this in detail.

\subsubsection{Study design}

To investigate whether the proposed methods improve the CAR experience of the TV show, we designed three experimental conditions: (1) basic trajectory, (2) summarization trajectory, and (3) additional summarization trajectory. The basic trajectory does not apply the proposed method and provides AR content by considering only the stories' sequence. The summarization trajectory provides the AR content by applying methods to reduce spatial transitions. The additional summarization trajectory provides additional AR content during the spatial transition in the summarization trajectory. We examined the proposed spatial transition management effect on CAR experiences by comparing and analyzing three trajectories. We conducted a between-subjects study to exclude the learning effects of the story and place. We adopted questionnaires used to measure cinematic virtual reality (CVR) experiences as both CAR and CVR users experience stories based on 3D environments (MacQuarrie and Steed 2017).

We expected that reducing spatial transition would lower the perceived workload and concern about missing something and increase narrative engagement, spatial presence, and system usability. We supposed that the additional

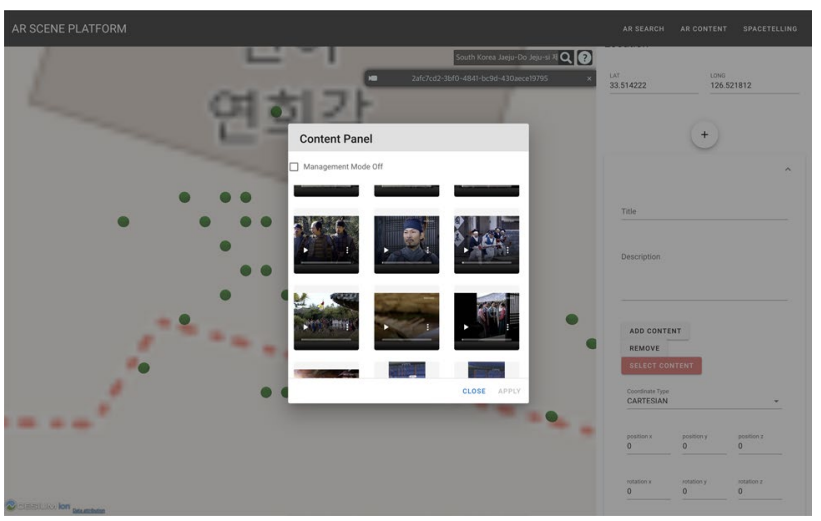

Fig. 2 Creating AR content of the TV show in the authoring tool

content in spatial transition would lower concern about missing something and improve narrative engagement, spatial presence, and system usability. However, we expected that providing additional content would increase the perceived workload because users have to view more content for a longer time in an outdoor environment. Finally, we expected that applying integrated spatial transition management would improve the overall CAR experience.

\subsubsection{System implementation}

To conduct user studies, we implemented the desktop-based authoring tool and mobile application. The authoring tool consists of two modes for managing the AR content and generating trajectories. As shown in Fig. 2, the AR content can be created by mapping the video, image, audio, and 3D models of the TV show scenes to the map. Authors can add various tags that signify the integrated context of the story to the AR content. They can also search for AR content based on their location and generate trajectories using them.

In generating trajectories, the recommendation list of the next content is provided based on the selected content when the author selects the first content of the global trajectory. Then, the author chooses the recommended content by determining the location of the local trajectory and the number of AR contents provided in each local trajectory according to the author's intentions, as shown in Fig. 3. Based on the selected content, the authoring tool provides the following list of recommended contents again. Throughout this process, the author can create a global trajectory of TV show series. The authoring tool was implemented by Vue Framework 2.6.10, and the interface was developed using Vuetify 2.1.14. Cesium, version 1.63.1, was used as a web-based 3D map. The server was implemented using Java 1.8 based Play Framework version 2.8.0, and the database used MariaDB 10.4 . 


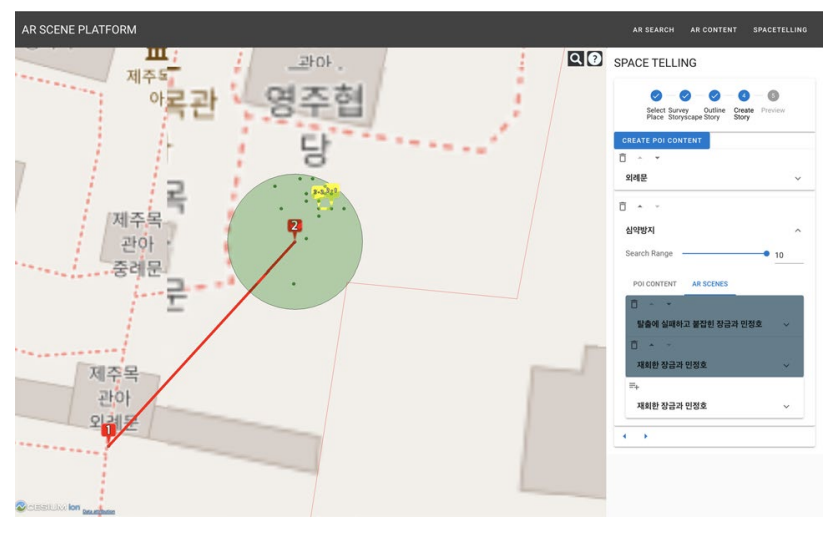

Fig. 3 Generating TV show trajectory in the authoring tool based on recommended content

The recommendation module for the AR content was implemented on the server side using the Java. We adopted an ontology approach for managing tag information of AR content. We applied the OWL API to create and modify the ontology model of $\mathrm{W}^{4}$. We also utilized the Geotools Java library to implement geospatial analysis to recommend additional AR content during the spatial transition. The recommended AR content position was the center point of the path line between two AR contents with a different place tag in the trajectory. The real path line was analyzed using the buffer and intersection geospatial analyses.

We designed and implemented a CAR application for mobile devices using Unity 2019.1.10f1. We used ARFoundation to track the position and orientation of the device (6-DoF) in a coordinate space. We implemented a global localization function using GPS and magnetic sensors to track the global position and orientation in real-world space. We additionally developed a manual calibration feature to improve the localization quality. This allowed users to check and perform localization on the 3D GIS map viewer. As shown in Fig. 5(a), in the viewer, users could gauge the localization quality by checking how well the actual buildings and the translucent cadastral 3D building models are aligned. If it is not correct enough, users can correct it by adjusting their location on the map viewer. We designed and implemented a UI to visualize information in a 3D outdoor space. We used iPhone XS MAX, iPhone 8 Plus, and iPhone $\mathrm{X}$ to use the latest ARKit capabilities in the user study. We are unable to publish our app because of copyright issues. However, we can provide a stimulus source when requested.

\subsubsection{Stimuli}

For the user study, we chose one of the most popular TV shows, called "Jewel in the Palace", which has led the filminduced tourism in Korea (Kim and Wang 2012; Kim 2012). We collected multimedia such as video clips and 3D models of the scenes with the background of the Jeju-mok government office ${ }^{4}$ where the heroine Jang Geum grew as a physician. The video content was used as the basic AR content that constitutes the TV show trajectory. The episode e and scene numbers are specified in the script. By comparing the script and video sources, it is possible to detect scene and episode changes. We edited the TV show by automatic shot detection of the video editing tool, Wondershare Filmora ${ }^{5}$. We manually merged them into a scene unit, referencing the script $^{6}$ based on the filming location. Finally, the video clips of 32 scenes were collected. To initialize the importance of the scenes, we collected the number of plays and likes of video clips in which the scene appeared on the official YouTube channel ${ }^{7}$ operated by the broadcasting company.

We created 3D content for four video scenes to provide a more immersive CAR experience. Each trajectory contained three 3D contents. We produced 3D models of the characters, background buildings, and props shown in the video scenes. To create a 3D face of an actor, we captured the image of actors from the video clip and reconstructed a face and a hairstyle using the Offline SDK of Avatar $\mathrm{SDK}^{8}$ in Unity 2019.1.10f1. We downloaded 3D body models wearing traditional Korean costumes from the Culture Content website $^{9}$ and modified them in Adobe Illustrator to match the texture or color represented in the TV show scene. Finally, we merged the face and body in Autodesk MAYA. We also created 3D models of the props and building using Autodesk 3D Max, referencing the captured image from the video.

In the authoring tool, we uploaded multimedia and registered each content at the corresponding location based on the map. Among the collected 32 scenes in the Jeju-Mok government office, nine scenes were excluded from registration as AR content because the relevant location was not accurate. In this process, the AR content includes proposed tags that describe the scene's context information and various spatial information such as position, rotation, and scale. In addition to "Jewel in the Palace," there were 314

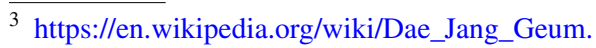

${ }^{4}$ http://www.jeju.go.kr/mokkwana/index.htm.

5 https://filmora.wondershare.com/.

${ }^{6}$ https://blog.daum.net/kehstudent/13620076.

7 https://www.youtube.com/channel/UCG5bAss12H0wjLG4BEv $5 \mathrm{ScQ}$.

8 https://avatarsdk.com/.

${ }^{9}$ http://www.culturecontent.com/main.do.
} 


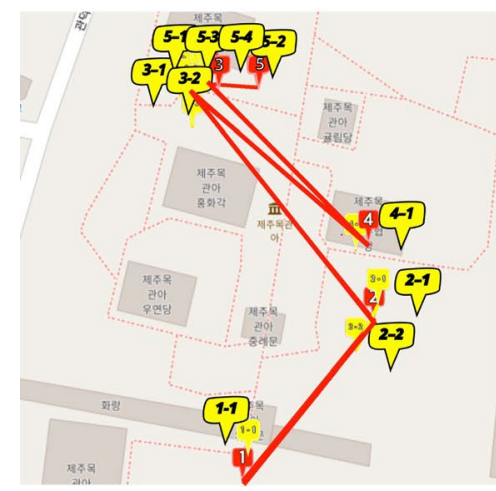

(a) Basic trajectory

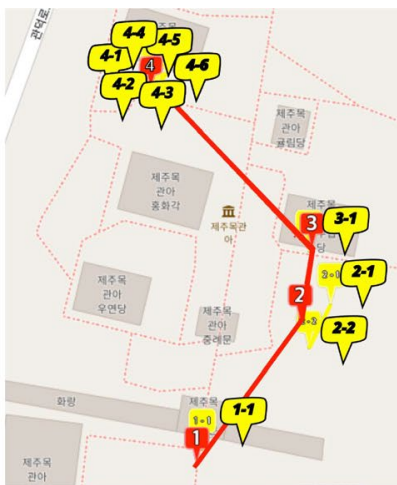

(b) Summarization trajectory

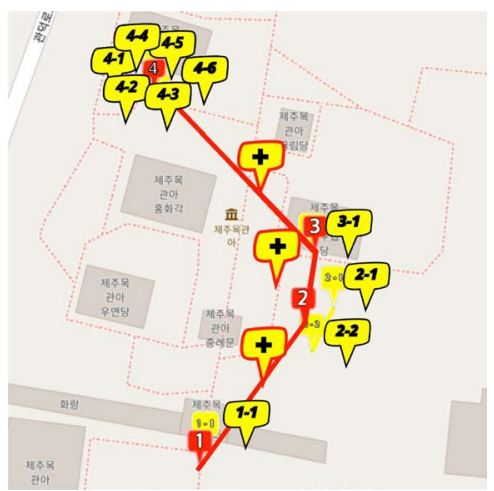

(c) Additional summarization trajectory

Fig. 4 The three experimental conditions

Table 2 AR content of each trajectory (BT: Basic Trajectory, ST: Summarization Trajectory, AST: Additional Summarization Trajectory)

\begin{tabular}{|c|c|c|c|c|c|c|c|}
\hline \multirow[b]{2}{*}{ BT } & \multicolumn{2}{|c|}{$\begin{array}{l}\text { Trajectory Condi- } \\
\text { tion }\end{array}$} & \multirow[t]{2}{*}{ Epi \# } & \multirow[t]{2}{*}{ Scene \# } & \multirow[t]{2}{*}{ Media Type } & \multirow[t]{2}{*}{ AR Content Title } & \multirow[t]{2}{*}{ Dur } \\
\hline & ST & AST & & & & & \\
\hline \multirow[t]{2}{*}{$1-1$} & $1-1$ & $1-1$ & 28 & 43 & VIDEO & Min Jeong-ho looking for Jang Geum & $0: 44$ \\
\hline & & + & - & - & VIDEO & Additional AR content & \\
\hline $2-1$ & $2-1$ & $2-1$ & 29 & 1 & VIDEO & Jang Geum caught after failing to escape & $1: 58$ \\
\hline $2-2$ & $2-2$ & $2-2$ & 29 & 3 & $3 \mathrm{D}$ & Min Jeong-ho who finally met Jang Geum & $1: 16$ \\
\hline $3-1$ & & & 29 & 57 & VIDEO & Jang Deok refusing royal physician position & $0: 51$ \\
\hline \multirow[t]{2}{*}{$3-2$} & & & 29 & 58 & $3 \mathrm{D}$ & Jang Geum deciding to become a royal physician & $0: 56$ \\
\hline & & + & - & - & VIDEO & Additional AR content & \\
\hline \multirow[t]{2}{*}{ 4-1 } & $3-1$ & $3-1$ & 31 & 13 & VIDEO & $\begin{array}{l}\text { Min Jeong-ho going on a business trip, Jang Geum remain- } \\
\text { ing alone }\end{array}$ & $1: 08$ \\
\hline & & + & - & - & VIDEO & Additional AR content & \\
\hline $5-1$ & $4-1$ & $4-1$ & 31 & 26 & VIDEO & The enemy seizing the office and confining the officials & $1: 14$ \\
\hline $5-2$ & $4-2$ & $4-2$ & 31 & 27 & $3 \mathrm{D}$ & Enemy looking for a doctor & $2: 41$ \\
\hline $5-3$ & $4-3$ & $4-3$ & 31 & 40 & VIDEO & Jang Geum successfully treating an enemy's chief & $0: 25$ \\
\hline \multirow[t]{3}{*}{$5-4$} & $4-4$ & $4-4$ & 31 & 44 & VIDEO & The enemy trying to leave with Jang Geum & $1: 15$ \\
\hline & $4-5$ & $4-5$ & 31 & 55 & VIDEO & Min Jung-ho Leaving for the capital & $1: 01$ \\
\hline & $4-6$ & $4-6$ & 31 & 58 & $3 \mathrm{D}$ & Jang Geum to be sent to the capital as a prisoner & $0: 39$ \\
\hline
\end{tabular}
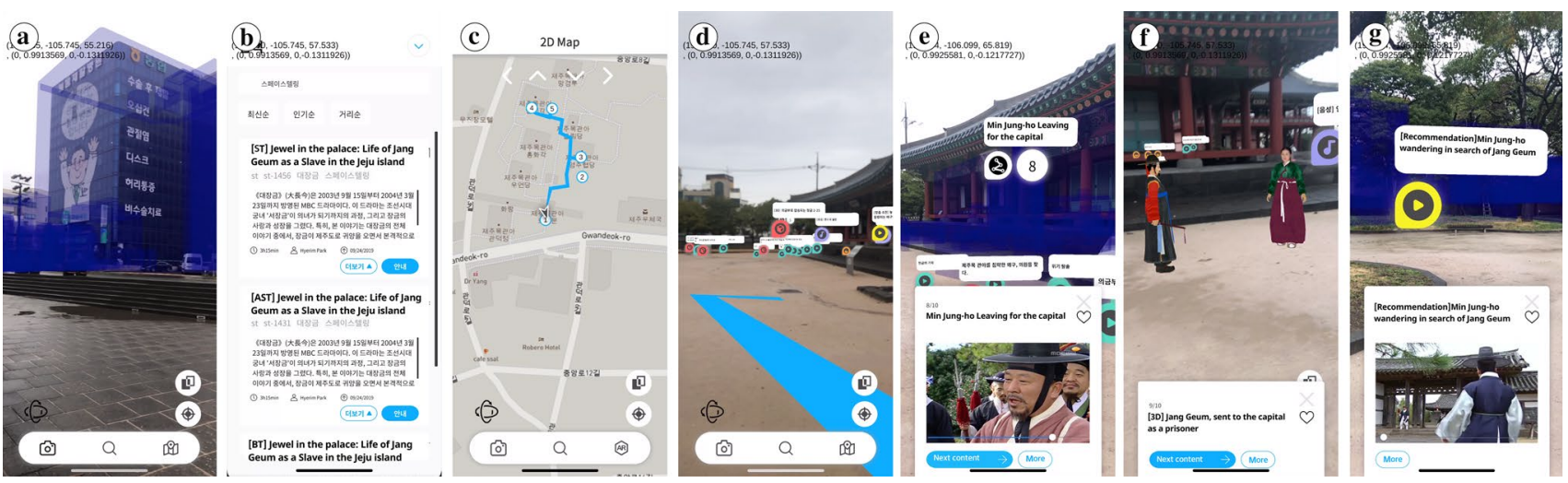

Fig. 5 CAR application: (a) tracking check, (b) search page, (c) 2D map, (d) AR navigation, (e) video content, (f) 3D content, (g) additional content 
AR contents with place tags for nine buildings in the JejuMok government office. This content included video, audio, image, and text of various TV shows and news filmed in or related to the Jeju-Mok government office. We used the play and like count and tag data of AR content to estimate the content importance and infer the user's interest.

\subsubsection{Setup}

Figure 4 shows the three experimental conditions and Table 2 shows all the AR contents included in each trajectory. In Fig. 4, as the AR content icons created in the authoring tool overlap and are not clearly visible, we additionally marked the AR content icon on the screen capture image of the authoring tool. In the AR content icon, the first number represents the order of the local trajectory, and the second number represents the order of the content in the local trajectory. For example, "4-1" refers to the first content viewed in the fourth local trajectory. The number indicated on the AR content icon in Fig. 4 and the number stated on the trajectory conditions in Table 2 represent the same AR content.

As shown in Fig. 4b, the summarization trajectory applied a method to reduce the spatial transition. This allowed users to view important TV show content sequentially within a simple tour path. The summarization trajectory of "Jewel in the Palace" was created with four local trajectories, including ten AR content. Specifically, there was one video content (1-1) at the Jeju-mok government office entrance. In the second local trajectory, there was one video content (2-1) and one 3D content (2-2). In the third local trajectory, there was one video clip (3-1). In the fourth local trajectory, there were four video clips (4-1, 4-3, 4-4, 4-5), and two 3D content (4-2, 4-6).

As shown in Fig. 4a, the basic trajectory was generated by considering only the episode and scene numbers. When the basic trajectory includes all AR content for 23 scenes of the TV show, there is a significant difference in the number of contents experienced in a place and experiment time. This can cause other factors to affect the experimental results in addition to spatial transition. Therefore, we first generated a global trajectory, including all the AR content. Then we controlled the number of content and media types in each local trajectory to be the same as the summarization trajectory taking into account only the scene order. As a result, the third and fifth local trajectories were in the same place, and participants had to visit the same site to view the content repeatedly.

As shown in Fig. 4c, the additional summarization trajectory provided an additional AR content in three spatial transitions in the summarization trajectory. Among the collected 32 scenes of the TV show, the summarization trajectory was authored with nine scenes. The other nine scenes were not uploaded to our database as AR content because it was difficult to identify the exact location. Therefore, our system provided additional content by applying the proposed method to the 14 remaining scenes. The additional trajectory finally included 13 AR contents, and the type of additional content included all videos.

\subsection{Measure}

We evaluated CAR experience of TV show trajectories, using five dependent variables: narrative engagement, concern about missing something, spatial presence, perceived workload, and system usability.

We measured the degree of engagement to the TV show's narrative through the Measuring Narrative Engagement Questionnaire (MNEQ) (Busselle and Bilandzic 2009). This scale examines how the participant engages with the story across four subscales: narrative understanding, attentional focus, narrative presence, and emotional engagement. We evaluated the narrative engagement with a total of 12 statements, three items per scale. All items were rated on a sevenpoint Likert scale.

We have adopted a questionnaire on the concern about missing something in the story (MacQuarrie and Steed 2017). This factor measures the user's concern about missing content when they experience narratives while moving in $3 \mathrm{D}$ space. We assessed this using two statements on a 5-point Likert scale.

The spatial presence was evaluated using the MEC Spatial Presence Questionnaire (Vorderer et al. 2004). The spatial presence has often been referred to as the experience of being present in a mediated environment (Heeter 1992). We evaluated the spatial presence using a total of 24 statements, four items per scale. All items were rated on a five-point Likert scale. In adopting the questionnaire, we replaced the "medium" with "TV show."

To assess the perceived workload users experience on the trajectory, we employed the NASA task load index (NASA TLX) (Hart and Staveland 1988). There are six subscales: mental demand, physical demand, temporal demand, performance, effort, and frustration. We adopted the "raw TLX" (Hart 2006) approach, and the scores were rated from 0 to 100 .

To rate system usability, we adopted the System Usability Scale(SUS) (Bangor et al. 2008). The questionnaire focuses on the experience of interacting with a wide range of systems designed to perform specific tasks. We used original SUS statements that are composed of 10 statements, scored on a five-point Likert scale.

\subsection{Procedure}

We experimented with the Jeju-mok government office, the actual background place of the TV show. The Institutional 
Review Board approved this study and its procedures. All participants signed consent forms regarding the study procedure and the data they provided. We registered the temporal user ID beforehand to record each user's movement or interaction with the AR content. When the participants arrived at the Jeju-mok government office entrance, we briefly introduced the experiment al process and TV show. The experimenter demonstrated how to use the application after confirming that the application's tracking module was running, as shown in Fig. 5a. Then, participants were asked to use the application and had time to familiarize themselves with the system.

After logging in with each participant's ID, we selected the trajectory randomly assigned to the participant on the search page, as shown in Fig. 5b. To see the user interest and disengagement, we informed the participants that it was possible to freely view other content if they were interested in them while experiencing the trajectory. Before starting the TV show trajectory experience, participants were able to see the entire route through the 2D map, as shown in Fig. 5c. Participants were able to switch the screen to the AR mode by pressing the AR button on the map page. The user could view the TV show AR content by touching the white icon, as shown in Fig. 5e. They also could watch the additional content by touching the yellow icon while transitioning, as shown in Fig. 5g.

In the TV show trajectory, participants experienced AR content such as video (Fig. 5e) and 3D models (Fig. 5f). They followed the AR navigation to see the next content, as shown in Fig. 5d. Our system temporarily saved data of AR content that participants played or liked in the user profile. This information was used to infer the user's interest and provide additional content. During the experiment, one assistant followed the participant by flying on the wall to deal with the participants' requests in using the system. The experiment's total time took about 30 minutes: 10 minutes for introducing the application and 20 minutes for experiencing the trajectory.

After participants completed the TV show trajectory experience, they answered eight questionnaires using the online Google Forms in the following order : (1) narrative engagement; (2) concern about missing something; (3) spatial presence; (4) NASA TLX; (5) System Usability Scale(SUS); (6) Domain-Specific Interest(DSI); (7) IRI fantasy scale; and (8) demographic information. Lastly, we conducted an open-ended interview for about 10 minutes to understand the user experience of the TV series trajectory. After the experiment, participants received approximately $\$ 15$ gift vouchers as compensation.

\subsection{Participants}

We recruited 24 experimental participants from the bulletin boards of universities on Jeju Island and websites for part-time jobs. The reason we recruited a relatively small number of experimental participants was as follows. Jeju Island, where we conducted the user study, is not only the country's most popular domestic tourist destination but is also especially popular for Chinese tourists. However, when we conducted the user study, the COVID-19 infection of Chinese tourists returning from their trip to Jeju Island was confirmed. As a result, the government suspended a visa-free program for foreign travelers to Jeju Island for the first time in history. At the same time, as social distancing and preventive measures were implemented for residents on the island, we had difficulties in recruiting participants for experiments. For this reason, we conducted a user study of 24 experimental participants.

To eliminate the possibility that significant differences in participants' domain interest and empathetic ability to the content may act as an underlying factor in the respondent's outcome, we measured domain-specific interest (DSI) (Vorderer et al. 2004) and the Interpersonal Reactivity Index (IRI) fantasy scale (Davis 1983). There were no outliers among the participants (DSI: $M=4.66, S D=.55$; IRI fantasy scale: $M=3.67, S D=1.04$ ) and no significant difference across the study groups [DSI: $F(2,21)=.377$, $p=.691$, IRI fantasy scale: $F(2,21)=.191, p=.827$ ]. Thus, the final sample consisted of eight participants for each of the three conditions.

Participants' ages ranged from 19 to 36 years $(M=$ $25.10, S D=4.603$ ), and of those, 12 were identified as female and 12 as male. The majority of the participants were not familiar with using AR application: 16 of them had never experienced the AR application, 6 of them had tried the AR application more than five times, and only two of them had used AR application often. Regarding the prior knowledge of the experiment place, 18 people had previously visited the Jeju-mok government office, but the rest of them had not visited it. Participants were also asked to rate the degree of the prior knowledge of the TV show on a 5-point Likert scale. One was denoted as "I have no idea about the TV show story" and five was denoted as "I know the TV show story very well." Most of them knew the title and outline of the TV show story, but not the details of the story because it had been released more than 10 years ago $(M=3.00, S D=1.609)$. There was no significant difference in the prior knowledge of the TV show between subject groups $[F(2,21)=.413, p=.667]$. 


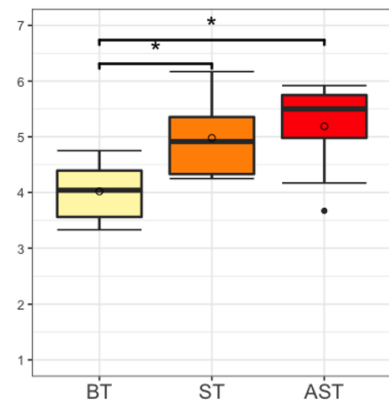

(a) Narrative engagement

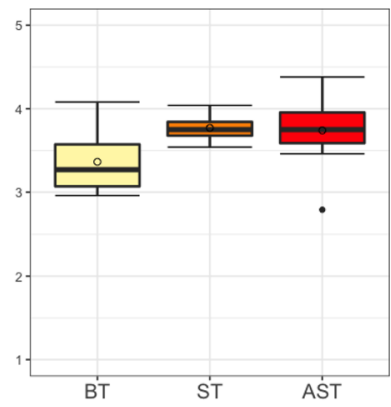

(e) Spatial presence

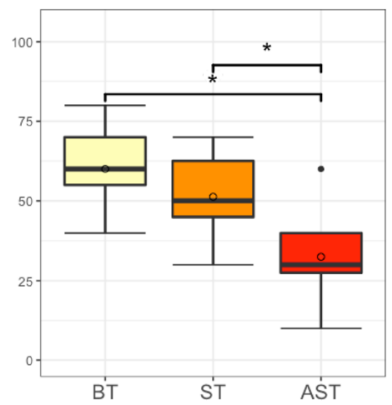

(i) Effort

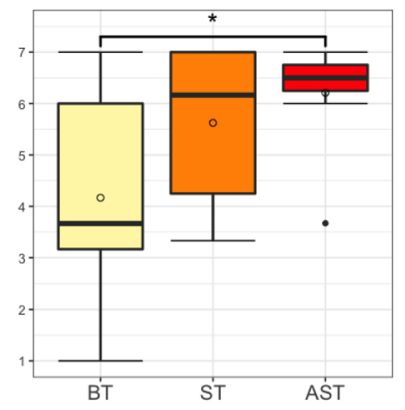

(b) Narrative understanding

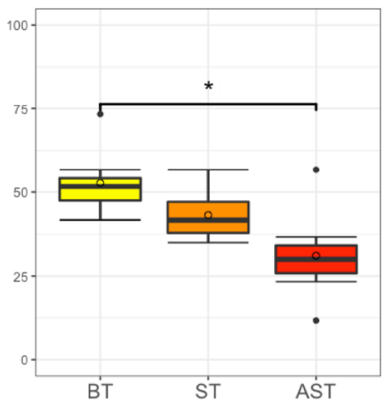

(f) Overall workload

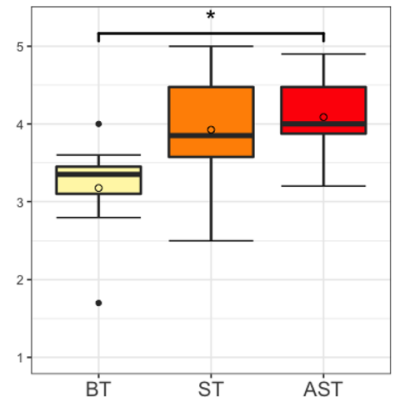

(j) System usability

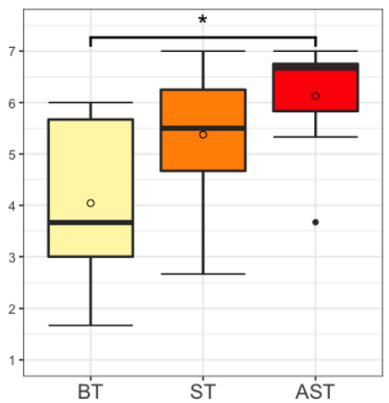

(c) Attentional focus

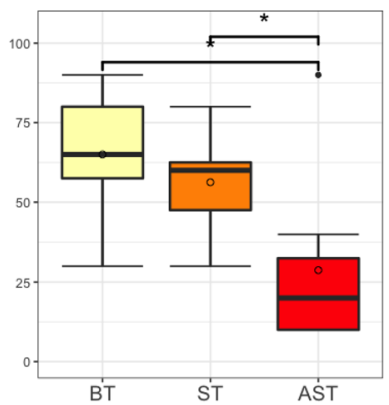

(g) Physical demand

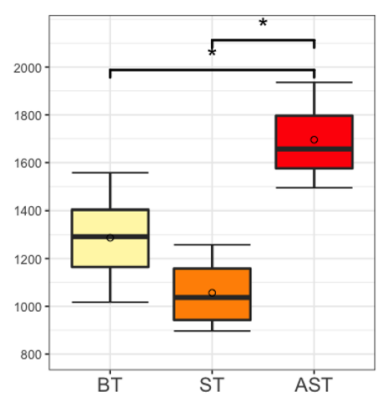

(k) Task completion time

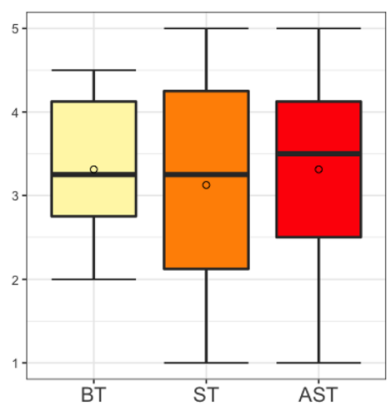

(d) Concern about missing something

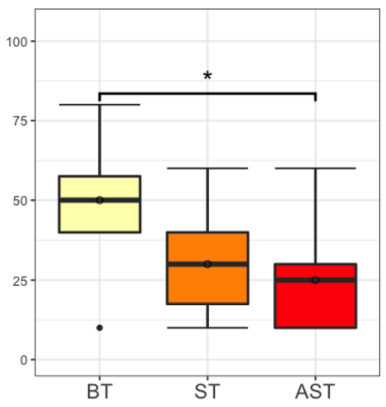

(h) Temporal demand

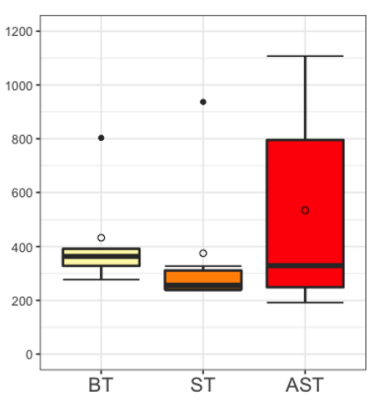

(l) Distance traversed

Fig. 6 The result of quantitative analysis about cinematic augmented reality experience for each study condition (BT: Basic Trajectory, ST: Summarization Trajectory, AST: Additional Summarization Trajectory), * $: p<.05$

\section{Results}

This section reports the results of a quantitative analysis of the five dependent variables and participants' movements for each TV program trajectory. We also describe the qualitative analysis results obtained from the participant interviews.

\subsection{Quantitative analysis}

We used one-way ANOVA for the quantitative results analysis for five dependent variables, including their subscales. We used the Shapiro-Wilk test to test the normality of the data distributions and confirmed that our data met the normality assumption. We also conducted Levene's test to examine the homogeneity of variance. We performed post hoc tests to determine the significant difference between experimental conditions. During the experiment, we stored the user's latitude and longitude positions every 3 seconds using the GPS sensor of the mobile device. Based on these data, we generated normalized heat maps representing the range of movement of the experimental participants and visualized the movement path. We investigated differences in the way participants viewed content in each trajectory through movement analysis. 


\subsubsection{Narrative engagement}

The different trajectories had a significant effect on the narrative engagement of the participants at the $p<.05$ level for the three conditions $[F(2,21)=6.555, p=.006]$. As shown in Fig. 6(a), post hoc comparisons using the Tukey HSD test indicated that the mean score for the summarization trajectory $(M=4.97, S D=.69)$ and the additional trajectory $(M=5.18, S D=.82)$ were significantly different from the basic trajectory $(M=4.01, S D=.51)$. However, the additional summarization trajectory did not significantly differ from the summarization trajectory.

There was a significant effect of the different trajectories on the narrative understanding of the participants at the $p<.05$ level for the three conditions $[F(2,21)=3.538$ $p=.047]$. As shown in Fig. 6b, post hoc comparisons using the Tukey HSD test indicated that the mean score for the additional summarization trajectory $(M=6.20, S D=$ 1.08) was significantly different from the basic trajectory $(M=4.16, S D=2.01)$. However, the summarization trajectory $(M=5.62, S D=1.50)$ did not significantly differ from the basic trajectory. The additional summarization trajectory also did not significantly differ from the summarization trajectory.

There was a significant effect of the different trajectories on the attentional focus of the participants at the $p<.05$ level for the three conditions $[F(2,21)=4.486, p=.024]$. As shown in Fig. 6c, post hoc comparisons using the Tukey HSD test indicated that the mean score for the additional summarization trajectory $(M=6.12, S D=1.13)$ was significantly different from the basic trajectory $(M=4.04, S D$ $=1.60)$. However, the summarization trajectory $(M=5.37$, $S D=1.43$ ) did not significantly differ from the basic trajectory. The additional summarization trajectory also did not significantly differ from the summarization trajectory.

\subsubsection{Concern about missing something}

There was no significant effect of the different trajectories on the concern about missing something of the participants at the $p<.05$ level for the three conditions $[F(2,21)=.053$, $p=.949$; the basic trajectory: $M=3.31, S D=.99$; the summarization trajectory: $M=3.12, S D=1.62$; the additional summarization trajectory: $M=3.31, S D=1.30]$.

\subsubsection{Spatial presence}

There was no significant effect of the different trajectories on the spatial presence of the participants at the $p<.05$ level for the three conditions $[(F(2,21)=2.880, p=.078)$; the basic trajectory: $M=3.36, S D=.37$; the summarization trajectory: $M=3.77, S D=.17$; the additional summarization trajectory: $M=3.74, S D=.50]$.

\subsubsection{Perceived workload}

There was a significant effect of the different trajectories on the perceived workload of the participants at the $p<.05$ level for the three conditions $[F(2,21)=9.156, p=.001]$. As shown in Fig. 6f, post hoc comparisons using the Tukey HSD test indicated that the mean score for the additional summarization trajectory $(M=31.04, S D=12.84)$ was significantly different from the basic trajectory $(M=52.70$, $S D=9.59)$. However, the summarization trajectory $(M=$ $43.12, S D=7.20$ ) did not significantly differ from the basic trajectory. The additional summarization trajectory also did not significantly differ from the summarization trajectory.

There was a significant effect of the different trajectories on the physical demand of the participants at the $p<.05$ level for the three conditions $[F(2,21)=6.344, p=.007]$. As shown in Fig. $6 \mathrm{~g}$, post hoc comparisons using the Tukey HSD test indicated that the mean score for the additional summarization trajectory $(M=28.75, S D=26.95)$ was significantly different from the basic trajectory $(M=65.00 S D$ $=19.27)$ and the summarization trajectory $(M=56.25, S D$ $=15.97)$. However, the summarization trajectory did not significantly differ from the basic trajectory.

There was a significant effect of the different trajectories on the temporal demand of the participants at the $p<.05$ level for the three conditions $[F(2,21)=3.769, p=.040]$. As shown in Fig. 6h, post hoc comparisons using the Tukey HSD test indicated that the mean score for the additional summarization trajectory $(M=25.00, S D=16.90)$ was significantly different from the basic trajectory $(M=50.00$, $S D=22.67)$. However, the summarization trajectory $(M=$ $30.00, S D=17.72$ ) did not significantly differ from the basic trajectory. The additional summarization trajectory also did not significantly differ from the summarization trajectory.

There was a significant effect of the different trajectories on the effort of the participants at the $p<.05$ level for the three conditions $[F(2,21)=7.151, p=.004]$. As shown in Fig. 6i, post hoc comparisons using the Tukey HSD test indicated that the mean score for the additional summarization trajectory $(M=32.50, S D=14.88)$ was significantly different from the basic trajectory $(M=60.00 S D=14.14)$ and the summarization trajectory $(M=51.25, S D=15.52)$. However, the summarization trajectory did not significantly differ from the basic trajectory.

\subsubsection{System usability}

There was a significant effect of the different trajectories on the system usability of the participants at the $p<.05$ level for the three conditions $[F(2,21)=3.734, p=.041]$. As shown 


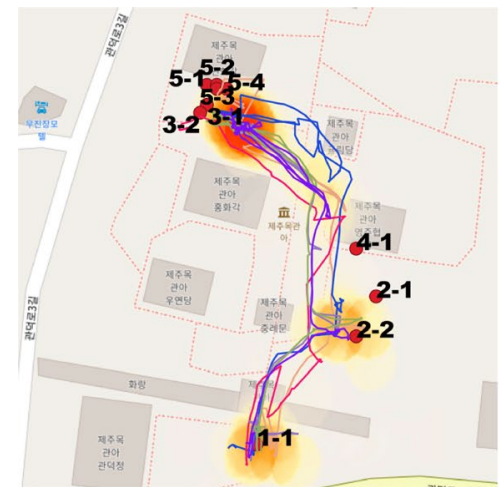

(a) Basic trajectory

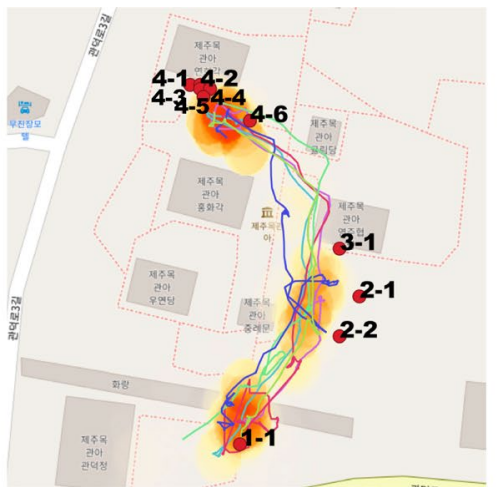

(b) Summarization trajectory

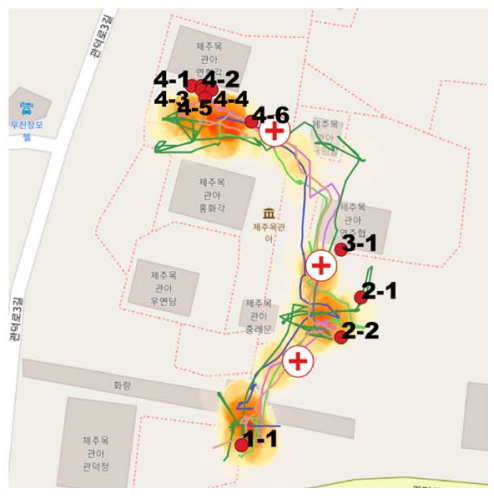

(c) Additional summarization trajectory

Fig. 7 Participants' movement in each TV show trajectory (path color: participant ID)

in Fig. 6j, post hoc comparisons using the Tukey HSD test indicated that the mean score for the additional summarization trajectory $(M=4.08, S D=.57)$ was significantly different from the basic trajectory $(M=3.17, S D=.68)$. However, the summarization trajectory $(M=3.92, S D=.85)$ did not significantly differ from the basic trajectory. The additional summarization trajectory also did not significantly differ from the summarization trajectory.

\subsubsection{Task completion time}

There was a significant effect of the different trajectories on the task completion time (sec) of the participants at the $p<.05$ level for the three conditions $[F(2,12)=12.252, p=$ .001]. As shown in Fig 6k, post hoc comparisons using the Scheffe test indicated that the mean score for the additional summarization trajectory $(M=1695.83, S D=223.09)$ was significantly different from the summarization trajectory $(M=1056.46, S D=145.63)$ and the basic trajectory $(M=$ $1286.91, S D=197.64)$. However, the summarization trajectory did not significantly differ from the basic trajectory.

\subsubsection{Distance traversed}

There was no significant effect of the different trajectories on the distance traversed $(\mathrm{m})$ of the participants at the $p<.05$ level for the three conditions $[F(2,13)=.378, p=.692$; the basic trajectory: $M=432.95, S D=211.31$; the summarization trajectory: $M=375.31, S D=277.19$; the additional summarization trajectory: $M=534.48, S D=399.22]$.

\subsubsection{Participant behavior}

In the basic trajectory, the third and fifth local trajectories were created in the same place called Yeonhui Gak, and the fourth local trajectory was authored in a place called Yeongju Hyupdang. Thus, in Fig. 7a, the movement paths show that the participants repeatedly went back and forth between Yeonhui Gak and Yeongju Hyupdang. In addition, the heatmap indicates that participants stayed longer where each local trajectory was authored to view the content. In particular, the participants spent the most time in Yeonhui Gak, where two local trajectories were provided and had the highest content. Furthermore, the heatmap shows that the participants viewed the authored content from the back of the building rather than the front in the fourth local trajectory. This reveals that when the content was not within the natural movement path, the participants consumed the content while minimizing the movement. In the spatial transition area, the participants did not stay and focused only on moving to the following local trajectories.

There are differences in participant motion and position in the second and third local trajectories in the summarization trajectory compared to the basic trajectory. In the basic trajectory, the heatmap shows that the participants did not move much in the second local trajectory. They stood in a confined area, watched the content, and immediately moved to the next place. In contrast, as shown in Fig. 7b, in the same place, the participants stayed in the broader area by successively viewing the contents of the local trajectories authored in two adjacent places. In addition, the participants viewed the contents of the third local trajectory in front of the building which is the scene's background. As with the basic trajectory, the participants stayed the most in the fourth local trajectory, which had the highest content, and did not stay in the spatial transition area.

Compared with other experimental conditions, as shown in Fig. 7c, participants stayed longer in the spatial transition areas in the additional summarization trajectory to view additional content. As a result, the participants experienced TV show content for a longer time in broader areas along the global trajectory, including the local trajectories and spatial transition areas. 
On the other hand, we could derive the typical behavioral patterns of viewing the content of the TV show trajectory. When experiencing the TV show trajectory, participants viewed the content primarily in the inner area between the full movement path and embedded content. As shown in Fig. 7, participants hardly explored the space behind where the TV show AR content was placed. Participants also showed different ways of viewing content depending on the content type. For example, the participants did not deviate significantly from the augmented navigation route when viewing the video. They clicked the content icon floating in the air and watched the video without much movement. However, when viewing 3D content, participants moved more actively to view the content up close or from multiple directions.

\subsection{Qualitative analysis}

We collected qualitative feedback through open-ended interviews to gain deeper insights into the proposed method. The coder classified the data according to each experimental condition. In the process of data classification, organization, and interpretation, researchers shared a note of comments involved in disagreements and different opinions. We organized the classification results as follows.

\subsubsection{Reducing the spatial transition}

Improvement in unnatural path The basic trajectory participants felt that the basic trajectory was unorganized, unnatural, and boring (BT1: "I hope that the route is not complicated but arranged.", BT5: "I felt uncomfortable and bored when I traveled the repeated path.", BT7: "It was not difficult to go back and forth, but it was not natural.").

However, they did not get frustrated when revisiting the same place under our experimental conditions. They stated that they did not feel that the route was physically and mentally challenging (BT2: "there was no physical difficulty in taking the route.", $\mathrm{BT} 3:$ "The route was not difficult but appropriate."). Some participants thought the trajectory was too simple, considering the size of the entire Jeju-mok government office and wanted to visit more buildings and experience diverse content (BT1: "It would have been nice if the route was made to move around more.", BT5: "The trajectory was simple, considering the size of the entire Jejumok government office. I want to explore more buildings, experiencing related diverse content.").

Participants in the other conditions said that the path was appropriate and not hard to explore physically (ST3: "The overall experience path was appropriate.", ST6: “It wasn't uncomfortable. It was new. It was okay to find the content while moving.", AST2: "The route was not difficult but appropriate.", AST4: "There was no physical difficulty in taking the route").

A better understanding of story To reduce spatial transition, the content importance was considered in creating the summarization trajectory, but not for the basic trajectory. Participants of the basic trajectory stated that they had difficulty in understanding and immersing in the story (BT2: "I thought it would not be easy to understand the narrative for anyone who does not know the contents of the drama.", BT4: "It was not easy to understand the story since I had watched the drama a long time ago. Anyone who has not seen the drama may be confused in understanding the content.").

The participants of the summarization trajectory did not experience much difficulty in understanding the story, although they found that there was some content missed in the trajectory (ST3: "I felt there was a missing story in the middle, but it was not difficult to understand the situation before and after the story.", ST5: "I could see that there was a missing story in the trajectory, but there was no difficulty in understanding it.").

\subsubsection{Filling the spatial transition}

A better understanding of the story The additional summarization trajectory participants said it was easy to understand the whole story (AST3: "The story was easy to understand. The recommended content was good for grasping the story.", AST4: "There was no problem in understanding the story of the TV series, and the recommendation content was satisfactory. Viewing the recommended video was not long. This made me understand the story well.", AST5: "I could grasp the story happened in Jeju-mok government office.").

\subsubsection{Overall outdoor CAR experience}

Preferred CAR trajectory Participants of the additional summarization trajectory considered the outdoor CAR experiences more positively compared with participants who experienced other trajectories (AST1: "I hope that the application is completed and commercialized. It was interesting to experience 'Jewel in the Palace' that I watched in the video at the background location. Through this experience, I felt that the site was more interesting. I really enjoyed this experience.", AST2: "Through augmented reality content, I felt the Jeju-mok government office is a fun and attractive place. I hope this content will be known to many people."). Participants of other conditions also said that it would be great content for visitors who like the TV series and visit background places or filming locations (ST1: "It would be great content for tourists to enjoy.”). Others said that a trajectory of historical TV shows in the heritage sites would be useful for educational purposes (BT1: "It was nice to 
be able to get closer to the heritage site using the TV show content.").

Preferred content type In terms of content type for CAR, we found that users are most fond of viewing 3D content. They felt like the 3D characters of the TV show were in the field through the AR view (BT3: "It was enjoyable to see $3 D$ models.", AST1: "when watching $3 D$ content in augmented reality, it was fun because the characters seemed to be in front of me."). In particular, most participants wanted the 3D model to move dynamically and interact with them (BT1: "I hope that the 3D model moves.", BT5: "If 3D models move and talk, it will feel like they are in a real environment.", ST4: "If the 3D model moves, the immersion will increase". AST5: "It would be better if I could interact with the $3 D$ model and the 3D model moves.").

Needs for the missing story We were concerned that participants should stand and watch the video clips in a winter outdoor environment. However, the participants did not feel that the video's length was long. (ST1: "If the story continues a little more, it will be more fun.", ST4: "The duration of the video was not that long.", ST5: "I did not care about the duration of the video."). Instead, some participants pointed out that short videos cut according to location interfered with immersion in the story. (ST4: "The episode was repeatedly interrupted by edit according to the location, so it was difficult to understand the content.", ST6: "The video was short and felt insufficient to be immersed to the story.").

\section{Discussion}

In this section, we analyze the study results and propose design implications for providing a better CAR experience.

\subsection{Analysis on the study results}

\subsubsection{The effect of reducing the spatial transition}

We considered the importance and location of AR content to reduce spatial transitions. In the summarization trajectory, the participants experienced higher narrative engagement than the basic trajectory. Both basic and summarization trajectories provided content in the order of the story, and we controlled the number and type of content experienced in the same place equally. The difference was that participants in the summarization trajectory viewed important content within a simple path that reduced spatial transition.

However, the task completion time, distance traveled, perceived workload, and its subscales, which we assumed to be associated with reduced spatial transitions, did not differ significantly between the two conditions. In addition, in interviews, participants of the basic trajectory stated that the route was unnatural and tedious but not physically demanding or challenging. Therefore, we interpreted that the participants' narrative engagement was improved by including important content rather than reducing the spatial transition.

In addition, the participants' movement data show that participants of the summarization trajectory watched TV show content at the author's intended location within the movement path. They explored the locations of the story more broadly and stayed longer by viewing the content in consecutive places. Thus, we can say that the TV show trajectory should consider the location of the content to deliver the CAR experience intended by the author within the user's natural path of travel.

\subsubsection{The effect of filling the spatial transition}

We hypothesized that the perceived workload would increase as the additional summarization trajectory's participants had to see more content in winter outdoor environments. However, the additional content in spatial transition decreased the physical demand and effort in terms of the perceived workload, although it increased task completion time. This implies that the participants perceived the trajectory as more comfortable and slack than the summarization trajectory's participants in physical demand. It also shows that they thought that experiencing the trajectory was easier than the summarization trajectory's participants in effort. In the interview, participants said it was easier to understand the story in the additional summarization trajectory. The quantitative result analysis showed an overall high narrative understanding with less variation in the additional summarization trajectory.

We considered order, importance, and user interest to provide additional content. However, when considering the order of scenes, the number of contents for recommendation in each spatial transition was small. Most participants focused on the TV show trajectory content and rarely played other content. Consequently, the additional content does not adequately reflect the user's interest. Thus, we interpreted that additional content considering content sequence and importance helped participants understand and be immersed in the story, leading to a decrease in the user's physical demand and effort. Also, while participants of other trajectories focused only on moving to the following local trajectory in spatial transitions, participants in the additional summarization trajectory stayed longer in the spatial transition area by viewing additional content.

\subsubsection{The effect of integral spatial transition management}

When comparing the basic trajectory and additional summarization trajectory, we found that the integrated spatial transition management enhanced the TV show's overall 
CAR experience. It increased narrative engagement and subscales such as narrative understanding and attentional focus. It also decreased the overall perceived workload and subscales, such as physical demand, temporal demand, and effort. It also increased the evaluation of the system usability. In particular, participants felt less time pressure in terms of temporal demand, although the task completion time was significantly longer. In the interview, the participants rated the additional summarization trajectory the most positively. Considering the effect of each spatial transition management, we interpreted it as follows. Providing more content within an organized path considering the importance of content increased the overall narrative engagement and decreased the perceived workload. In addition, these experiences led participants to evaluate the CAR system highly.

On the other hand, there was no significant difference in concern about missing something and spatial presence in the three trajectories. Concern about missing something is caused by the freedom of movement and gaze when the user experiences scattered story pieces in a 3D space. However, we guided the participants exactly where to view the content through AR navigation. Thus, there was no significant difference between the conditions.

We expected that the proposed method would enhance the spatial presence of the TV show story's background location. However, we did not observe any significant differences between the experimental conditions. In the TV show trajectory, participants followed the AR navigation to a designated location and viewed the drama's story set in the areas. Balakrishna et al. suggested that the high guidance and high narrative condition for narrative transportation lower spatial presence (Balakrishnan and Sundar 2011). The reason is that as players focus their interest narrowly on the given task, they limit cognitive resources that can process spatial information or cues about the surrounding environment. In the participants' movement data, most participants stayed between AR navigation and content under all conditions and rarely explored the surrounding environment. Thus, we can say that as all three TV show trajectories had high navigation and high narrative function, there was no significant difference in spatial presence between trajectories.

\subsection{Design implications}

Based on our findings, we propose design implications to improve the authoring tool and user application for a better cinematic trajectory experience.

Integrating a location-based video content database In the currently developed authoring tool, authors should manually create AR content by mapping the video, image, audio, and $3 \mathrm{D}$ content of TV show scenes to the map. However, manually generating the data can reduce accessibility to creating trajectories and cause errors in the data itself. Thus, authors should quickly search drama or movie scenes based on location and use various media related to each scene. For this, we should build a location-based video content database by adopting various techniques such as geotagging or geoparsing, automatic metadata generation of the scenes, and reconstruction of 3D models from 2D videos.

In recognizing the narrative space, geotagging or geoparsing can be performed using related data such as scripts and call sheets as well as camera positions of the video (Leidner and Lieberman 2011). For the automatic metadata generation of scenes, we can use content recognition, automated tagging, and topic model learning (Kim et al. 2018b). Along with various text data such as scripts, captions, and call sheets, these technologies can provide information about the elements that make up the video scene, such as time, location, characters, props, special effects, and background music.

Various studies have been conducted to reconstruct 3D models from 2D videos (Russell et al. 2014; Jeni et al. 2015). It is now possible to reconstruct a dynamic 3D model of a person from a single RGB stream in real time (Alldieck et al. 2019; Habermann et al. 2019; Mehta et al. 2020). When the database built with these technologies is integrated into the authoring tool, it can efficiently support searching, visualizing, and manipulating various media types of content based on a map. This makes it easier to create and share cinematic trajectories.

Content recommendation with various options that can satisfy the author's intention The trajectory considering content importance improved the participants' narrative engagement and lowered the perceived workload. In a trajectory considering content location, participants saw the content at the position intended by the author within their natural movement path and more widely explored the site of local trajectories. Therefore, the authoring tool should support authors in designing trajectories, including important content, within their natural path of travel based on consecutive PoIs.

To recommend content in consideration of a natural movement path, it should consider not only the distance between AR content but also the direction of the content. In recommending important content based on consecutive PoIs, there can be various factors that authors consider important when designing a trajectory in addition to the content importance we proposed. Therefore, it is necessary to support content filtering according to various options such as actors and themes as well as the number of plays and likes of AR content. Through this, authoring tools can support creating a trajectory that meets the author's intentions.

Creating dynamic interaction of $3 D$ content for narrative space expansion Most participants of TV show trajectories preferred 3D content, and they wanted the 3D models to move dynamically and interact with them. In addition, when 
viewing $3 \mathrm{D}$ content, participants moved more actively in the background places to view the content. Thus, we can enhance participants' narrative engagement and lower the perceived workload by using dynamic and interactive 3D content for scenes. It is also possible to increase the spatial presence by inducing the user to explore the background space of the content by using 3D content. Authors can design interactions between visitors and avatars using placement, traversal, and formation rules (Singh et al. 2021). In addition, the author can create new narrative spaces with prequels, sequels, and spin-off secondary characters by expanding the time, place, and characters of the TV show's narrative space (Pratten 2011). This allows visitors to actively explore the story world while interacting with characters or objects and to experience location-based new stories that have not been released on TV or in movies before.

Marking the priority of the scene for adaptive trajectory guidance Enhancing the spatial presence of participants involves increasing the degree of freedom in content and place exploration. When passively experiencing trajectories created by authors, participants do not fully recognize and explore their surroundings as a narrative world. They focus solely on seeing the content contained in the trajectory. Therefore, users should be given the option to freely explore not only the content included in the trajectory but also other content not included in the trajectory. However, visitors may not have enough time to view the content of the trajectory when they are getting off the trajectory or viewing other content. Considering this, the authors can indicate the priority of a scene when creating a trajectory, just as Squeezebox adds priority semantics to segmented linear content (Evans et al. 2017). Through this, visitors can be guided to view content that should not be missed on the trajectory in consideration of the audience's tour context such as the remaining tour time or current location.

Simulation in a mirror world Currently, in authoring tools, the author can see the created trajectory on the map but cannot simulate how the visitor experiences the trajectory. Thus, the author should be able to dynamically simulate the trajectory from the visitor's point of view before publishing the cinematic trajectory. The preview can be implemented as a mirror world with a 3D virtual city model linked to various internal and external databases (Du et al. 2019; Kukka et al. 2017). Through realistic and immersive simulation, the authors can create and modify a cinematic trajectory more efficiently.

Content recommendation in spatial transition area based on location-based video summarization The spatial transition area does not play a role as a narrative world because users simply focus on moving when content is not provided. To utilize the entire space in which the global trajectory is created as a narrative world, additional content should be continuously provided in the spatial transition area. Thus, we propose to provide additional content of TV shows based on location-based video summarization.

Video summarization is typically performed to satisfy properties such as conciseness, representativeness, and informativeness (Ji et al. 2019). Various studies have been conducted on video summarization for movies and TV series (Irie et al. 2010; Hesham et al. 2018). Based on the location-based video content database, we can perform location-based video summarization, including scenes of the area where the trajectory is created. Among scenes in location-based video summarization, the content can be recommended by considering the essential or impactful scenes (Cao et al. 2020) and user interests (McKercher and Du Cros 2003; Tsai and Huang 2018). Through this, we can realize a complete narrative space in the spatial transition area and allow users to experience a more immersive narrative world.

\subsection{TV show trajectory generalizability: applicable regions and strategies}

The generalizability of the proposed TV show trajectory is summarized as follows, focusing on the places where it can be applied and the method of providing content.

We can apply our method to a place with a movie map that allows people to visit the filming location on foot (Beeton 2006). For example, in the UK, the USA, and Hong Kong, hundreds of maps provide information on where certain scenes have been filmed and where stars shop, eat and stay. Among these maps are various walking tours. For instance, a 2-hour walking tour program among the Harry Potter movie maps allows people to visit more than ten filming locations from the film series in downtown London ${ }^{10}$. Applying the proposed AR experience in these areas is expected to provide a more immersive narrative experience.

It is also possible to apply TV show trajectories to small and rural communities where filming locations are concentrated (Beeton 2008). In particular, when feature films and drama series are filmed in a non-urban area, large-scale open sets are built, or filming takes place in various locations around the main location. In this case, as there is a filming location within a short distance, our method is applicable.

TV show trajectories can be provided in theme parks that reproduce the spatial background of movies and dramas. In particular, some theme parks reconstruct the spatial background of the narrative without considering the audience's movement in terms of experiencing the content. There are also theme parks that do not properly provide content or programs that allow visitors to

\footnotetext{
$\overline{10}$ https://www.visitbritainshop.com/us/en/brit-movie-tours-wizardslondon-walking-tour-harry-potter-film-locations.
} 
experience the drama story (Kim and Nam 2016). In these places, an interactive trajectory can provide various story experiences, consistently attracting visitors.

The historical drama content we used is easy to understand and share with its nationals. It often has the theme of a common opinion or attitude about the purposes or values of a nation or ethnicity. However, due to the diverse background of the audience or the nature of the content genre, only viewing the essential scenes related to the location may make it difficult for the audience to grasp the narrative or its fictional world. Therefore, to apply the proposed method, various prior knowledge levels of the audience about the content should be considered. The audience should be able to see sufficient pre-contents before starting the trajectory and freely view the content of interest while exploring the spatial background.

\subsection{Limitations and future work}

We did not clearly show the effect of reducing the number of spatial transitions in this study. However, from the user study, we found that participants wanted to visit as many different places as possible while experiencing the story. Therefore, in future work, it will be necessary to optimize the spatial transition within the trajectory by considering the context of the user and the real environment, rather than simply reducing the number of spatial transitions or distances. We specifically investigate how the spatial transition affects the user's CAR experience according to the degree of pre-engagement of tourists with content, walkability, and authenticity of the real environment of the TV show trajectory.

We could not adequately reflect user interest in providing additional content. Accurate user interest estimation is important when recommending additional content during spatial transitions and interactively adjusting trajectories according to the user's content and spatial exploration. Therefore, in future research, we will propose a method to estimate user interests through real-time behavioral data, including interactions with AR content.

Finally, we applied the proposed method to a TV show. We used one of the most popular TV shows, and participants had a general perception of the TV show's characters or overall plot. We expect our experimental results to be applicable to other content with similar prior knowledge levels by CAR users. However, in future work, by applying our proposed method to various TV shows, we will generalize the experimental results and demonstrate the practicality of the TV show trajectory.

\section{Conclusion}

We proposed spatial transition management to provide a cinematic experience of a TV show based on the real world. We reduced the spatial transition by considering the sequence, location, and importance of TV show content when creating a TV show trajectory. We filled the spatial transition with additional TV show content taking into account the order and importance of content and user interests. The user study results showed that reducing spatial transition increases narrative engagement and the additional content in spatial transition decreases physical demand and effort. Integrated spatial transition management increased the overall narrative engagement, perceived workload, and system usability. The user study results demonstrated that the proposed spatial transition management significantly enhanced CAR experience of the TV show.

Our study makes the following contributions. Most studies on AR television (ARTV) are limited to living rooms or indoor environments where viewers conventionally watch TV. They also only introduce prototypes or do not conduct systematic user studies. However, to validate our proposed method, we performed a large-scale outdoor environment modeling. We implemented a system that can provide TV show content in an AR environment by utilizing contextual information of users and content. We conducted a systematic user study. Based on our findings, we presented the design implications of the authoring tool and mobile application for future research. As a result, this study extended the ARTV concept outdoors by proposing and verifying how to provide virtual TV content in an augmented outdoor world (Vatavu et al. 2020)

Funding This work was supported by the Institute of Information \& Communications Technology Planning \& Evaluation (IITP) grant funded by the Korea government(MSIT) (No.2019-0-01270, WISE AR UI/UX Platform Development for Smartglasses).

This research is supported by Ministry of Culture, Sports and Tourism and Korea Creative Content Agency(Project Number: R2021080001).

\section{Declarations}

Ethics approval This study and all procedures were approved by the Korea Advanced Institute of Science and Technology (KAIST) Institutional Review Board (KH2020-011).

\section{References}

Alldieck T, Pons-Moll G, Theobalt C, Magnor M (2019) Tex2shape: Detailed full human body geometry from a single image. In: Proceedings of the IEEE/CVF International Conference on 
Computer Vision, pp 2293-2303, https://doi.org/10.1109/ICCV. 2019.00238

Balakrishnan B, Sundar SS (2011) Where am i? how can i get there? impact of navigability and narrative transportation on spatial presence. Hum Comput Interact 26(3):161-204. https://doi.org/ 10.1080/07370024.2011.601689

Bangor A, Kortum PT, Miller JT (2008) An empirical evaluation of the system usability scale. Int J Hum-Comput Interact 24(6):574-594. https://doi.org/10.1080/10447310802205776

Beeton S (2006) Community development through tourism. Landlinks Press, https://doi.org/ 10.1137/1.9781611972832.442

Beeton S (2008) Location, location, location: Film corporations' social responsibilities. J Travel Tour Mark 24(2-3):107-114. https://doi. org/10.1080/10548400802092551

Benford S, Giannachi G (2008) Temporal trajectories in shared interactive narratives. In: Proceedings of the SIGCHI Conference on Human Factors in Computing Systems, pp 73-82, https://doi.org/ 10.1145/1357054.1357067

Benford S, Giannachi G, Koleva B, Rodden T (2009) From interaction to trajectories: designing coherent journeys through user experiences. In: Proceedings of the SIGCHI Conference on Human Factors in Computing Systems, pp 709-718, https://doi.org/10. 1145/1518701.1518812

Benford S, Crabtree A, Flintham M, Greenhalgh C, Koleva B, Adams M, Tandavanitj N, Farr JR, Giannachi G, Lindt I (2011) Creating the spectacle: Designing interactional trajectories through spectator interfaces. ACM Trans Comput-Human Interact (TOCHI) 18(3):1-28. https://doi.org/10.1145/1993060.1993061

Busselle R, Bilandzic H (2009) Measuring narrative engagement. Media Psychol 12(4):321-347. https://doi.org/10.1080/15213 260903287259

Cao C, Shi Z, Yu M (2020) Automatic generation of diegetic guidance in cinematic virtual reality. In: 2020 IEEE International Symposium on Mixed and Augmented Reality (ISMAR), IEEE, pp 600-607, https://doi.org/10.1109/ISMAR50242.2020.00087

Caquard S, Fiset JP (2014) How can we map stories? a cybercartographic application for narrative cartography. J Maps 10(1):18-25. https://doi.org/10.1080/17445647.2013.847387

Davis MH (1983) Measuring individual differences in empathy: Evidence for a multidimensional approach. J Person Soc Psychol 44(1):113. https://doi.org/10.1037/0022-3514.44.1.113

Dow S, Mehta M, Lausier A, MacIntyre B, Mateas M (2006) Initial lessons from ar façade, an interactive augmented reality drama. In: Proceedings of the 2006 ACM SIGCHI international conference on Advances in computer entertainment technology, pp 28-es, https://doi.org/10.1145/1178823.1178858

Du R, Li D, Varshney A (2019) Geollery: a mixed reality social media platform. In: Proceedings of the 2019 CHI Conference on Human Factors in Computing Systems (CHI), CHI, p 13, https://doi.org/ $10.1145 / 3290605.3300915$

Evans M, Ferne T, Watson Z, Melchior F, Brooks M, Stenton P, Forrester I, Baume C (2017) Creating object-based experiences in the real world. SMPTE Motion Imaging J 126(6):1-7. https://doi.org/ 10.5594/JMI.2017.2709859

Fosh L, Benford S, Reeves S, Koleva B, Brundell P (2013) See me, feel me, touch me, hear me: trajectories and interpretation in a sculpture garden. In: Proceedings of the SIGCHI conference on human factors in computing systems, pp 149-158, https://doi.org/ $10.1145 / 2470654.2470675$

Gao H, Tang J, Hu X, Liu H (2015) Content-aware point of interest recommendation on location-based social networks. In: Aaai, vol 15, pp 1721-1727, https://doi.org/ 10.1137/1.9781611972832.442

Ghellal S, Morrison A, Hassenzahl M, Schaufler B (2014) The remediation of nosferatu: exploring transmedia experiences. In: Proceedings of the 2014 conference on Designing interactive systems, pp 617-626, https://doi.org/10.1145/2598510.2600881
Habermann M, Xu W, Zollhoefer M, Pons-Moll G, Theobalt C (2019) Livecap: Real-time human performance capture from monocular video. ACM Trans Graph (TOG) 38(2):1-17. https:// doi.org/10.1145/3311970

Hansen FA, Kortbek KJ, Grønbæk K (2012) Mobile urban drama: interactive storytelling in real world environments. New Rev Hypermed Multimed 18(1-2):63-89. https://doi.org/10.1080/ 13614568.2012.617842

Hargood C, Hicks B, Charles F, Lynch S, Tang W (2017) Snow white is missing: An interactive locative story for dementia patients. In: International Conference on Technologies for E-Learning and Digital Entertainment, Springer, pp 85-92, https://doi.org/ 10.1007/978-3-319-65849-0_10

Hargood C, Weal MJ, Millard DE (2018) The storyplaces platform: Building a web-based locative hypertext system. In: Proceedings of the 29th on Hypertext and Social Media, pp 128-135, https://doi.org/10.1145/3209542.3209559

Hart SG (2006) Nasa-task load index (nasa-tlx); 20 years later. In: Proceedings of the human factors and ergonomics society annual meeting, Sage Publications Sage CA: Los Angeles, CA, vol 50, pp 904-908, https://doi.org/10.1177/154193120605000 909

Hart SG, Staveland LE (1988) Development of nasa-tlx (task load index): Results of empirical and theoretical research. In: Advances in psychology, vol 52, Elsevier, pp 139-183, https://doi.org/10. 1016/S0166-4115(08)62386-9

Heeter C (1992) Being there The subjective experience of presence. Presence Teleop Virt Environ 1(2):262-271. https://doi.org/10. 1162/pres.1992.1.2.262

Hesham M, Hani B, Fouad N, Amer E (2018) Smart trailer: Automatic generation of movie trailer using only subtitles. In: 2018 First International Workshop on Deep and Representation Learning (IWDRL), IEEE, pp 26-30, https://doi.org/10.1109/IWDRL.2018. 8358211

Hunter J, Armstrong L (1999) A comparison of schemas for video metadata representation. Comput Netw 31(11-16):1431-1451. https://doi.org/10.1016/S1389-1286(99)00053-5

Irie G, Satou T, Kojima A, Yamasaki T, Aizawa K (2010) Automatic trailer generation. In: Proceedings of the 18th ACM international conference on Multimedia, pp 839-842, https://doi.org/10.1145/ 1873951.1874092

Jeni LA, Cohn JF, Kanade T (2015) Dense 3d face alignment from 2d videos in real-time. In: 2015 11th IEEE international conference and workshops on automatic face and gesture recognition (FG), IEEE, vol 1, pp 1-8, https://doi.org/10.1109/FG.2015.7163142

Ji Z, Xiong K, Pang Y, Li X (2019) Video summarization with attention-based encoder-decoder networks. IEEE Trans Circuits Syst Video Technol 30(6):1709-1717. https://doi.org/10.1109/TCSVT. 2019.2904996

Kim H, Bang S, Woo W (2018a) Authoring personal interpretation in a 3d virtual heritage site to enhance visitor engagement. In: 2018 3rd Digital Heritage International Congress (DigitalHERITAGE) held jointly with 2018 24th International Conference on Virtual Systems \& Multimedia (VSMM 2018), IEEE, pp 1-8, https://doi. org/10.1109/DigitalHeritage.2018.8810093

Kim S (2012) A cross-cultural study of on-site film-tourism experiences among chinese, japanese, taiwanese and thai visitors to the daejanggeum theme park, south korea. Curr Issues Tour 15(8):759-776. https://doi.org/10.1080/13683500.2011.640394

Kim S, Nam C (2016) Hallyu revisited: Challenges and opportunities for the south korean tourism. Asia Pac J Tour Res 21(5):524-540. https://doi.org/10.1080/10941665.2015.1068189

Kim S, Wang H (2012) From television to the film set: Korean drama Daejanggeum drives Chinese, Taiwanese, Japanese and Thai audiences to screen-tourism. Int Commun Gaz 74(5):423-442. https:// doi.org/10.1177/1748048512445152 
Kim SH, Jung D, Lee SY, Kim SJ (2018b) Scene-based metadata generation and open api provisioning method for smart broadcast service. In: 2018 20th International Conference on Advanced Communication Technology (ICACT), IEEE, pp 664-668, https:// doi.org/10.23919/ICACT.2018.8323875

Kukka H, Pakanen M, Badri M, Ojala T (2017) Immersive street-level social media in the $3 \mathrm{~d}$ virtual city: Anticipated user experience and conceptual development. In: CSCW, pp 2422-2435, https:// doi.org/10.1145/2998181.2998341

Leidner JL, Lieberman MD (2011) Detecting geographical references in the form of place names and associated spatial natural language. Sigspatial Spec 3(2):5-11. https://doi.org/10.1145/20472 96.2047298

Liest $\varnothing 1 \mathrm{G}$ (2014) Along the appian way. storytelling and memory across time and space in mobile augmented reality. In: Euro-Mediterranean Conference, Springer, pp 248-257, https://doi.org/10.1007/ 978-3-319-13695-0_24

MacIntyre B, Bolter JD, Vaughan J, Hannigan B, Moreno E, Haas M, Gandy M (2002) Three angry men: dramatizing point-of-view using augmented reality. In: ACM SIGGRAPH 2002 conference abstracts and applications, pp 268, https://doi.org/10.1145/12420 73.1242281

MacIntyre B, Bolter JD, Vaughn J, Hannigan B, Gandy M, Moreno E, Haas M, Kang SH, Krum D, Voida S (2003) Three angry men: An augmented-reality experiment in point-of-view drama. In: Proc. International Conference on Technologies for Interactive Digital Storytelling and Entertainment (TIDSE'03), Citeseer, pp 230-236, http://citeseerx.ist.psu.edu/viewdoc/download?doi = 10.1.1.9.6233\&rep $=$ rep $1 \&$ type $=$ pdf

MacQuarrie A, Steed A (2017) Cinematic virtual reality: Evaluating the effect of display type on the viewing experience for panoramic video. In: Virtual Reality (VR), 2017 IEEE, IEEE, pp 45-54, https://doi.org/10.1109/VR.2017.7892230

McKercher B, Du Cros H (2003) Testing a cultural tourism typology. Int J Tour Res 5(1):45-58

Mehta D, Sotnychenko O, Mueller F, Xu W, Elgharib M, Fua P, Seidel HP, Rhodin H, Pons-Moll G, Theobalt C (2020) Xnect: Realtime multi-person $3 \mathrm{~d}$ motion capture with a single rgb camera. ACM Trans Graph (TOG) 39(4):82-1. https://doi.org/10.1145/ 3386569.3392410

Mine MR, Van Baar J, Grundhofer A, Rose D, Yang B (2012) Projection-based augmented reality in disney theme parks. Computer 45(7):32-40. https://doi.org/10.1109/MC.2012.154

Naliuka K, Carrigy T, Paterson N, Haahr M (2010) A narrative architecture for story-driven location-based mobile games. In: International Conference on Web-Based Learning, Springer, pp 11-20, https://doi.org/10.1007/978-3-642-20539-2_2

Nisi V, Costanza E, Dionisio M (2017) Placing location-based narratives in context through a narrator and visual markers. Int Comput 29(3):287-305

Packer HS, Hargood C, Howard Y, Papadopoulos P, Millard DE (2017) Developing a writer's toolkit for interactive locative storytelling. In: International Conference on Interactive Digital Storytelling, Springer, pp 63-74, https://doi.org/10.1007/978-3-319-71027-3_ 6)

Park H, Woo W (2015) Metadata design for location-based film experience in augmented places. In: Mixed and Augmented RealityMedia, Art, Social Science, Humanities and Design (ISMARMASH'D), 2015 IEEE International Symposium on, IEEE, pp 40-45, https://doi.org/10.1109/ISMAR-MASHD.2015.12

Park H, Woo W (2017) Metadata design for ar spacetelling experience using movie clips. In: Consumer Electronics (ICCE), 2017 IEEE
International Conference on, IEEE, pp 388-391, https://doi.org/ 10.1109/ICCE.2017.7889365

Park H, Kim E, Kim H, Shin Je, Kim J, Kim K, Woo W (2018a) K-culture time machine: A mobile ar experience platform for korean cultural heritage sites. In: International Conference on Human Interface and the Management of Information, Springer, pp 167180, https://doi.org/10.1007/978-3-319-92046-7_15

Park H, Kim J, Bang S, Woo W (2018b) The effect of applying filminduced tourism to virtual reality tours of cultural heritage sites. In: 2018 3rd Digital Heritage International Congress (DigitalHERITAGE) held jointly with 2018 24th International Conference on Virtual Systems \& Multimedia (VSMM 2018), IEEE, pp 1-4, https://doi.org/10.1109/DigitalHeritage.2018.8810089

Pratten R (2011) Getting started with transmedia storytelling. CreateSpace

Russell C, Yu R, Agapito L (2014) Video pop-up: Monocular 3d reconstruction of dynamic scenes. In: European conference on computer vision, Springer, pp 583-598, https://doi.org/10.1007/ 978-3-319-10584-0_38

Shin Je, Park H, Woo W (2017) Connecting the dots: Enhancing the usability of indexed multimedia data for ar cultural heritage applications through storytelling. In: Proceedings of the 15th International Workshop on Content-Based Multimedia Indexing, pp 1-6, https://doi.org/10.1145/3095713.3095725

Singh A, Kaur R, Haltner P, Peachey M, Gonzalez-Franco M, Malloch J, Reilly D (2021) Story creatar: a toolkit for spatially-adaptive augmented reality storytelling. In: 2021 IEEE Virtual Reality and 3D User Interfaces (VR), IEEE, pp 713-722, https://doi.org/10. 1109/VR50410.2021.00098

Spierling U, Kampa A (2014) Structuring location-aware interactive narratives for mobile augmented reality. In: International Conference on Interactive Digital Storytelling, Springer, pp 196-203, https://doi.org/10.1007/978-3-319-12337-0_20

Torchin L (2002) Location, location, location: The destination of the manhattan TV tour. Tour Stud 2(3):247-266. https://doi.org/10. $1177 / 14687976020023002$

Tsai CH, Huang JY (2018) Augmented reality display based on user behavior. Comput Stand Int 55:171-181. https://doi.org/10.1016/j. csi.2017.08.003

Tung WF (2015) Augmented reality for mobile service of film-induced tourism app. In: Mobile Services for Toy Computing, Springer, pp 129-140, https://doi.org/10.1007/978-3-319-21323-1_7

Vatavu RD, Saeghe P, Chambel T, Vinayagamoorthy V, Ursu MF (2020) Conceptualizing augmented reality television for the living room. In: ACM International Conference on Interactive Media Experiences, pp 1-12, https://doi.org/10.1145/3391614.3393660

Vorderer P, Wirth W, Gouveia FR, Biocca F, Saari T, Jäncke L, Böcking S, Schramm H, Gysbers A, Hartmann T, et al. (2004) Mec spatial presence questionnaire. Retrieved Sept 18:2015, https:// academic.csuohio.edu/kneuendorf/frames/MECFull.pdf

Wither J, Allen R, Samanta V, Hemanus J, Tsai YT, Azuma R, Carter W, Hinman R, Korah T (2010) The westwood experience: connecting story to locations via mixed reality. In: 2010 IEEE International Symposium on Mixed and Augmented Reality-Arts, Media, and Humanities, IEEE, pp 39-46, https://doi.org/10.1109/ ISMAR-AMH.2010.5643295

Publisher's Note Springer Nature remains neutral with regard to jurisdictional claims in published maps and institutional affiliations. 